\title{
Dealing with pre-existing chronic neutropenia in cancer patients- considerations and consequences in the clinical praxis
}

\author{
Anna-Birgitte Thinggaard ${ }^{1}$, Gabor Liposits ${ }^{1 a}$ (iD) and Niels Fristrup ${ }^{1,2}$ \\ ${ }^{1}$ Department of Oncology, Regional Hospital West Jutland, Denmark \\ ${ }^{2}$ Department of Oncology, Aarhus University Hospital, Denmark \\ ahttps://orcid.org/0000-0002-8204-3949
}

\begin{abstract}
Chronic neutropenia is a rare but important challenge with substantial clinical implications for patients receiving antineoplastic treatment. Treatment-induced neutropenia is a well-known adverse event during chemotherapy and some targeted treatments. Guidelines for administering chemotherapy are rather strict to protect the patient from severe and life-threatening complications. Consequently, patients with chronic neutropenia may receive suboptimal antineoplastic treatment. Autoimmune neutropenia or chronic idiopathic neutropenia (CIN) may affect the antineoplastic treatment by causing delayed drug delivery, dose reductions and early discontinuation of treatment. CIN is characterised by the onset in late childhood or adulthood, affects mostly women, is clinically benign and has rare spontaneous remission. Here, we elucidate the challenges related to chronic neutropenia when administering chemotherapy through two clinical cases. Guidelines may need to be revised in order to optimise the treatment of patients with asymptomatic chronic neutropenia, thus personalising the medical decisions for each patient.
\end{abstract}

Keywords: chronic neutropenia, autoimmune neutropenia, chronic idiopathic neutropenia, chemotherapy, targeted therapy, monoclonal antibodies

\section{Introduction}

Neutropenia is characterised by abnormally low levels of neutrophil granulocytes (further neutrophils) in the blood. The neutrophils play an important role in the immune response and neutropenia is known to increase the risk of serious infections [1]. The normal neutrophil count is defined between 2 and $7 \times 10^{\%} / \mathrm{L}$, with levels below $1.5 \times 10^{\circ} / \mathrm{L}$ defined as clinically relevant neutropenia [1]. Neutropenia is a well-known adverse event related to chemotherapy and consequently increases the risk of infection during treatment in patients with cancer.

Neutropenia is divided into three categories depending on the severity of the condition: mild $\left(>1 \times 10^{9} / \mathrm{L}\right)$, moderate $\left(0.5-1 \times 10^{9} / \mathrm{L}\right)$ and severe neutropenia $\left(<0.5 \times 10^{9} / \mathrm{L}\right)$ [2] .

Correspondence to: Niels Fristrup Email: niels.fristrup@rm.dk

ecancer 2020, 14:1131

https://doi.org/10.3332/ecancer.2020.1131

Published: $29 / 10 / 2020$

Received: $11 / 06 / 2020$

Publication costs for this article were supported by ecancer (UK Charity number 1176307).

Copyright: (c) the authors; licensee ecancermedicalscience. This is an Open Access article distributed under the terms of the Creative Commons Attribution License (http:// creativecommons.org/licenses/by/3.0), which permits unrestricted use, distribution, and reproduction in any medium, provided the original work is properly cited. 
Febrile neutropenia is a potential life-threatening adverse event related to systemic antineoplastic treatments (Table 1), leading to hospital admission, and may cause delay in treatment administration, and result in either dose reduction or treatment withdrawal, or addition of granulocyte colony-stimulating factor (G-CSF) [3]. Prior to chemotherapy administration, new haematological tests are required to ensure appropriate bone marrow function. The administration of chemotherapy or other drugs inducing neutropenia requires a certain level of neutrophils and thrombocytes, although it varies among treatment regimens [4]. If these requirements are not met, the treatment is contraindicated and will be postponed until the bone marrow function recovers.

An example of non-chemotherapy drugs inducing neutropenia is cyclin-dependent kinase 4/6 inhibitors (CDK4/6i). These targeted agents lead to cell cycle arrest (G1-S phase) [5]. This in turn leads to senescent-like cellular phenotype in the tumour cells [6]. A common adverse event related to the administration of CDK4/6i is severe neutropenia [5].

The prevalence of neutropenia in Caucasians is estimated being less than $1 \%$ and chronic neutropenia approximately $0.1 \%$ [7, 8]. Chronic neutropenia is classified as a neutrophil count $<1.5 \times 10^{9} / \mathrm{L}$ that persists for more than 3 months [9]. The condition spans widely; it occurs in all ages and may be insignificant or life-threatening. A range of extrinsic and intrinsic factors may cause chronic neutropenia, e.g., bone marrow dysfunction or malnutrition/starvation [9]. Table 2 summarises the factors or conditions that are a common cause for selective neutropenia, when neutropenia is first diagnosed and proven persistent in both time and multiple blood tests.

If no obvious reason for the neutropenia can be found, a bone marrow biopsy is usually recommended. Only if all tests are normal, 'idiopathic neutropenia' can be diagnosed $[9,10]$.

Table 1. Anti-cancer drugs leading to neutropenia.

\begin{tabular}{|c|c|}
\hline Drug type & Examples \\
\hline \multicolumn{2}{|l|}{ Targeted agents } \\
\hline Monoclonal antibodies & Rituximab (CD20), daratumumab (CD38), infliximab (TNF), venetoclax (BCL2) \\
\hline Cyclin-dependent kinase $4 / 6$ inhibitors & Palbociclib, ribociclib, abemaciclib \\
\hline Protein kinase inhibitors & Ibrutinib \\
\hline PARP inhibitors & Olaparib, niraparib, rucaparib, veliparib \\
\hline \multicolumn{2}{|l|}{ Chemotherapy } \\
\hline Antimetabolites & 5-flourouracil, methotrexate, capecitabine, gemcitabine \\
\hline Alkylating agents & Cyclophosphamide, carboplatin, oxaliplatin, cisplatin, lomustine \\
\hline Anti-tumour antibiotics & Doxorubicin, epirubicin, mitomycin C \\
\hline Topoisomerase inhibitors & Irinotecan, topotecan, etoposide \\
\hline Plant alkaloids & Vinorelbine, paclitaxel, docetaxel, etoposide, irinotecan, topotecan \\
\hline
\end{tabular}

Table 2. Haematological examination of potential conditions/causes of chronic neutropenia.

\begin{tabular}{|l|}
\hline Current symptoms and history (including use of drugs, malnutrition, comorbidity, recent travels or primary haematological malignancy) \\
\hline Concomitant symptoms \\
\hline Specific blood test to exclude rheumatological or haematological conditions \\
\hline Demographic factors \\
\hline Social details \\
\hline Past medical history (ethnicity, frequent hospitalisation as a child, recent infections, etc.) \\
\hline
\end{tabular}


Chronic idiopathic neutropenia (CIN) and autoimmune neutropenia (AIN) are often interchangeable in adults since no reliable antibody test is yet available [1]. AIN is characterised by antineutrophil antibodies circulating in the blood stream. It can be idiopathic (primary) or secondary. Secondary AIN is caused by some other underlying (autoimmune) disease. CIN is not associated with other pathologies. CIN is characterised by the onset in late childhood or adulthood, affects more women than men, is clinically benign and has rare spontaneous remission, whereas primary AIN is more frequent in children and often self-limiting $[9,11]$.

Here, we present two cases of chronic neutropenia in colon cancer (case \#1) and ovarian cancer (case\# 2) and highlight the dilemma of suboptimal anti-neoplastic treatment.

Colorectal cancer (CRC) is the third most common cause of cancer-related deaths in women [12].

Capecitabine (an oral prodrug of 5-fluorouracil) and 5-fluorouracil have long been the backbone of chemotherapy for stages II-III colon cancer in the adjuvant setting [13]. The duration of treatment has been proven most efficient when given for six months [14]. Haematological treatment requirements are neutrophils $\geq 1.5 \times 10^{\%} / \mathrm{L}$ and thrombocytes $\geq 100 \times 10^{\circ} / \mathrm{L}$.

Ovarian cancer is the eight most common cause of cancer-related deaths in women [12]. Mortality rates and survival depend greatly on age and are especially poor in women +70 -year old [15].

Carboplatin, doxorubicin and paclitaxel are among the most effective cytotoxic agents used for the systemic treatment of ovarian cancer [16]. For carboplatin in combination with doxorubicin, the haematological treatment requirements are neutrophils $\geq 1.5 \times 10^{9} / \mathrm{L}$ and thrombocytes $\geq 100 \times 10^{\%} / \mathrm{L}$, and for weekly paclitaxel neutrophils $\geq 1.5 \times 10^{9} / \mathrm{L}$ and thrombocytes $\geq 50 \times 10^{9} / \mathrm{L}$.

Table 3 shows the estimated risk of febrile neutropenia related to doxorubicin/carboplatin, paclitaxel/carboplatin, capecitabine/5-FU and paclitaxel alone.

The estimates may vary depending on the treatment regimen (dose $/ \mathrm{m}^{2}$, weekly administration (adm) vs three weekly (q3w), age, comorbidity, treatment intention and performance status [18].

During intermediate or high-risk treatments, added treatment with myeloid growth factor or G-CSF may be necessary. As a growth factor, G-CSF stimulates the genesis of white blood cells. This is especially important during adjuvant treatment in order to maintain dose intensity in case of persisting neutropenia or as a prophylaxis for febrile neutropenia [18]. Side effects are rarely severe and mostly consist of flu-like symptoms $[9,18]$. Treatment with G-CSF is rarely used when the patient is being treated with capecitabine since it has been suggested that the combination may increase the myelotoxicity of capecitabine through the proliferative activity of the bone marrow [19].

Ovarian cancer treatment with poly (ADP-ribose) polymerase inhibitors (PARPi), such as olaparib, is approved as maintenance therapy in platinum-sensitive breast cancer gene (BRCA)-mutated relapsed ovarian cancer [20]. Olaparib is administered as capsules consumed twice daily (maximum dosage $400 \mathrm{mg} \times 2$ ) on a continuous basis. Every treatment cycle is 28 days and requires neutrophils $\geq 1.5 \times 10^{9} / \mathrm{L}$ and thrombocytes $\geq 75 \times 10^{9} / \mathrm{L}[21]$.

Table 3. Risk of febrile neutropenia varies among anti-cancer drugs.

\begin{tabular}{|l|l|c|}
\hline \multicolumn{1}{|c|}{ Regimen } & \multicolumn{1}{|c|}{ Estimated risk of febrile neutropenia } & References \\
\hline Doxorubicin/carboplatin & Low $(\leq 10 \%)$ & {$[17,18]$} \\
\hline Paclitaxel/carboplatin & Low $(\leq 10 \%)$ & {$[17,18]$} \\
\hline Capecitabine/5-FU & Low to intermediate $(<20 \%)$ & {$[17,18]$} \\
\hline Paclitaxel alone & High $(>20 \%)$ & {$[17,18]$} \\
\hline
\end{tabular}


Table 4. Treatment overview case \#1.

\begin{tabular}{|l|l|}
\hline Intended treatment & $\begin{array}{l}\text { Capecitabine (1,000 } \mathrm{mg} / \mathrm{m}^{2} \text { twice daily) } 1,500 \mathrm{mg}(100 \%) \text { day 1-14 q3w, } \\
8 \text { series in total }\end{array}$ \\
\hline Final treatment & Capecitabine 1,150 $\mathrm{mg}$ (75\%) day 1-14, 6 series in total \\
\hline Postponements due to low neutrophil count & 11 times in total (each lasting 1 week) \\
\hline
\end{tabular}

\section{Case 1}

A 57-year-old woman, diagnosed with left-sided colon cancer in 2018 and with synchronous liver metastasis, was referred to the Department of Oncology, Regional Hospital West Jutland, for adjuvant chemotherapy after surgical removal of both primary tumour and liver metastasis.

The woman had no concomitant diseases and took no medication at time of referral.

The patient was candidate to adjuvant treatment with capecitabine. However, due to unexplained mild neutropenia, the start of the chemotherapy was postponed several times, fewer treatments were administered and the dose was reduced (Table 4). The patient was never admitted with febrile neutropenia. The patient was referred to the Department of Haematology in November 2019 for diagnostic work-up. Myelodysplastic syndrome (MDS) was excluded as a reason for the neutropenia. Figure 1 shows the level of neutropenia from the date of surgery (November 2018) until treatment was terminated (October 2019).

\section{Case 2}

A 67-year-old woman, diagnosed with FIGO stage IIIC BRCA-mutated high-grade serous ovarian adenocarcinoma in 2016, underwent macroscopic radical surgery, including hyperthermic intraperitoneal chemotherapy. She was referred to the Department of Oncology, Regional Hospital West Jutland, in order to receive adjuvant chemotherapy.

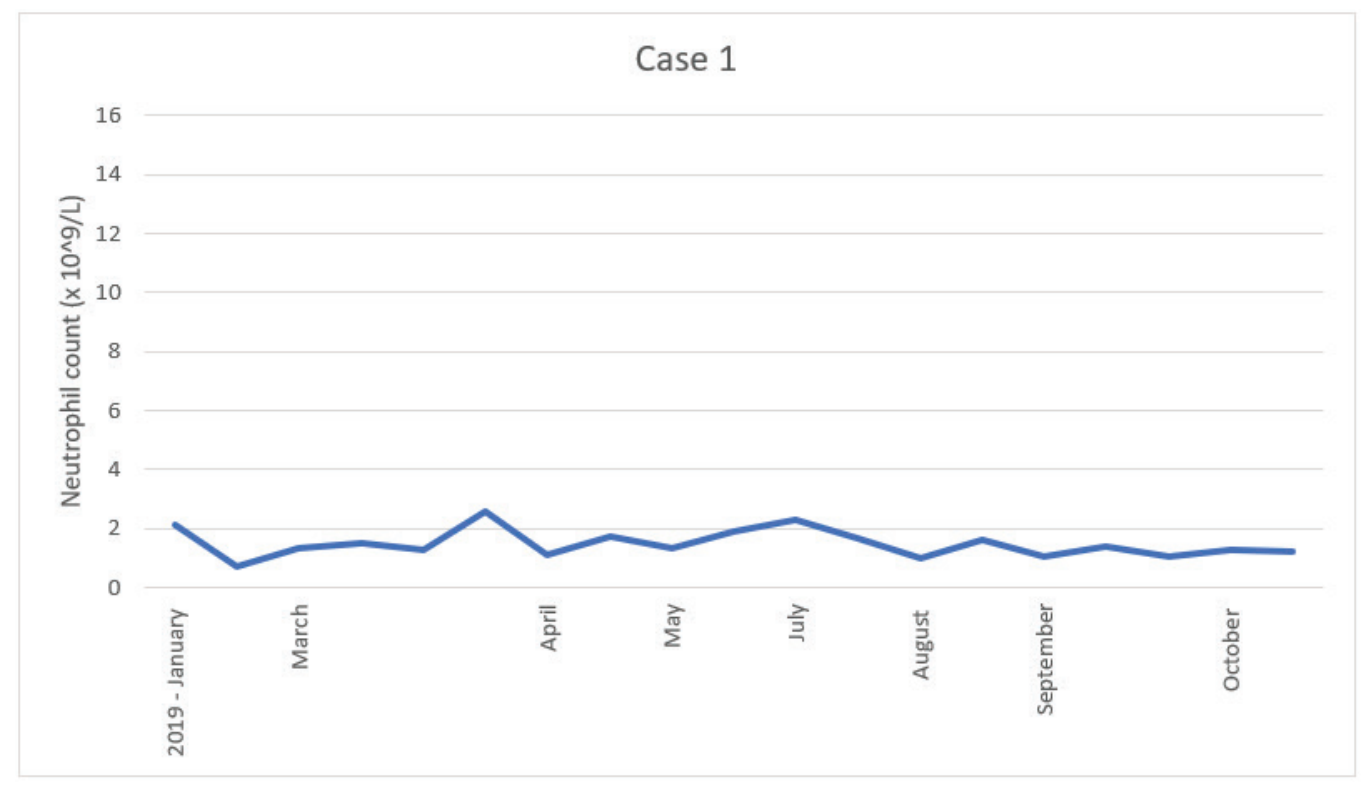

Figure 1. Illustration of the neutrophil count since cancer surgery was performed. 
The patient had no concomitant diseases except for allergy-induced asthma that required no daily medication.

Due to chronic moderate neutropenia even before chemotherapy initiation, treatment was postponed several times and the dose was significantly reduced despite addition of G-CSF (Table 5).

In the palliative setting, the patient became candidate for olaparib treatment; it was started, but stopped after only two cycles due to progression and persistent neutropenia.Later in the treatment course, a weekly paclitaxel regime with reduced requirements to the neutrophil count $\left(>1.0 \times 10^{\circ} / \mathrm{L}\right)$ was administered, but treatments were further postponed and dose-reduced several times despite lower neutrophils were accepted.All along the treatment course, the patient reported general well-being and no episode of fever. Figure 2 shows the level of neutropenia since surgery was performed and up until November 2019 when the patient, due to a decline in her general condition, was treated with corticosteroids daily, and future data were therefore no longer valid.

In September 2018, the patient was referred to the Department of Haematology and a bone marrow biopsy was carried out. MDS was excluded as a reason for the neutropenia. The bone marrow was described as hypoplastic but not dysplastic. Human immunodeficiency virus-testing was negative.

\begin{tabular}{|l|l|}
\hline Table 5. Treatment overview \#2. & \multicolumn{2}{l|}{$\begin{array}{l}\text { Paclitaxel 287 } \mathrm{mg} \text { (100\%) and carboplatin 640 mg (AUC 5) q3w, six series } \\
\text { in total }\end{array}$} \\
\hline Intended treatment & $\begin{array}{l}\text { Carboplatin } 609 \mathrm{mg} \text { (AUC 5) + G-CSF (Longuex 6 mg) q3w, 3-6 series in } \\
\text { total, and then treatment with Olaparib 400 } \mathrm{mg} \times 2 \text { (100\%) q4w* }\end{array}$ \\
\hline Planned treatment after relapse & $\begin{array}{l}\text { Olaparib terminated after only two series. Hereafter: } \\
\text { Paclitaxel 77 mg (60\%) weekly administered }\end{array}$ \\
\hline Pdministered treatment & 16 times in total (each lasting 1 week) \\
\hline${ }^{*}$ At this time, only Olaparib capsules were available & \\
\hline
\end{tabular}

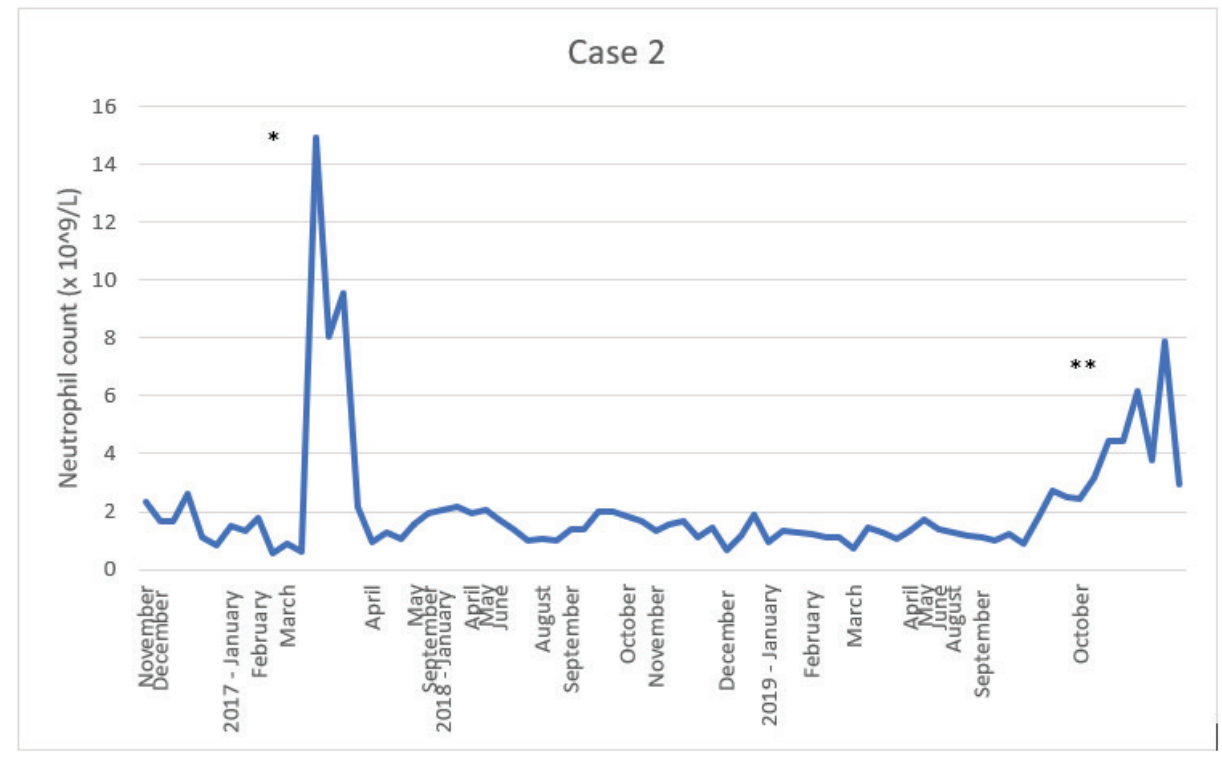

Figure 2. Illustration of the neutrophil count since cancer surgery was performed. ${ }^{*}$ Increase in neutrophil count to $14.92 \times 10^{\%} / \mathrm{L}$ due to one treatment with G-CSF (Longuex $6 \mathrm{mg}$ ) -no chemotherapy was given. ${ }^{* *}$ Increase in neutrophil count due to hospital admittance and treatment with corticosteroids. 
The Department of Pharmacology was contacted in November 2019 to investigate whether the neutropenia could be drug-induced, but no plausible explanation was found. Treatment has since been terminated due to the decline of the patient's general condition (January 2020).

\section{Discussion}

Optimal treatment with chemotherapy relies entirely on intervals and dose. If dose intensity is compromised, then treatment efficacy is assumed being suboptimal.

If the intervals are prolonged due to neutropenia, then there is a risk of less effective chemotherapy. This phenomenon is demonstrated in the 'fractional kill' and 'log cell kill' hypothesis [22]. Likewise, if neutropenia results in a lower dosage of chemotherapy, then the risk of insufficient antineoplastic treatment is higher. In line with this, we believe that the treatment with non-cytotoxic drugs is sub-optimal if postponements and dose reductions are implemented due to neutropenia.

Chemotherapy guidelines clearly state that no person should be treated with chemotherapy if the neutrophils are below a predefined level. The guidelines therefore do not take individual patient characteristics into consideration and leave no room for individual medical assessment.

In both cases presented here, the neutropenia was present even before chemotherapy was initiated and gave neither patient any symptoms. The other cell lines remained normal. These patients may have had asymptomatic neutropenia for years. In both cases, it was not possible to maintain dose intensity due to unexplained neutropenia, possibly resulting in less effective treatment. Furthermore, both patients had a markedly delayed initiation of treatment due to neutropenia when they were first referred to the Department of Oncology.

No particular reason for neutropenia was explained in the two clinical cases and many conditions may lead to chronic neutropenia.Cancer, chemotherapy (and other drugs), infections, metabolic disorders or vitamin deficiencies among other factors can cause neutropenia [9].

Conditions such as cyclic neutropenia, AIN and CIN are rare and difficult to diagnose [23].

Cyclic neutropenia is a particularly rare condition in which the neutrophil count fluctuates between abnormal and normal values. Episodes of neutropenia would happen every 2-5 weeks [23]. This is not the case for the two patients here, as the level of neutropenia has not changed over time.

Since the condition in both clinical cases presented here had been present for more than 3 months and other apparent reasons had been excluded, CIN or AIN seems more likely. CIN seems to match both cases in terms of age frequency, gender distribution and chronic tendency. However, AIN cannot be excluded here since none of the cases was tested for autoimmune antibodies. In AIN, neutrophils rise after steroid treatment due to a reduction in the destruction and a glucocorticoid-induced demargination, which is consistent with case \#2 (Figure 2).

Different suggestions in terms of treatment of the neutropenia can be speculated but requires thorough investigation with regard to the cause. Treatment could consist of immunoglobulin or methotrexate depending on the underlying reason for the condition. However, these treatments are usually only recommended when the patient have symptoms due to the neutropenia, which was not the case here. The treatment combination of chemotherapy and methotrexate can also be an issue.

CIN/AIN is the most plausible explanation to the neutropenia described in the above-mentioned cases. The objective of this article, however, is not to diagnose the underlying condition that caused the neutropenia in the first place, but to illustrate the complexity of dealing with such patients within the framework of the current guidelines.

The question that remains is whether asymptomatic patients with unexplained neutropenia (due to CIN or AIN) should be treated under the same restrictions as other patients or whether it would be appropriate for an individual clinical judgment to deviate from regulations in cases such as these to avoid insufficient antineoplastic treatment.

\section{Conclusion}

In conclusion, dose intensity is crucial for many antineoplastic treatments, and current guidelines leave no room for personalised treatment of cancer patients with chronic neutropenia. Furthermore, when patients present with unexplained neutropenia before cancer treatment 
initiation, a haematological examination should be carried out quickly to avoid unnecessary postponement of the cancer treatment initiation. We still know little about how to treat chronic neutropenia in cancer patients, and whether it should be treated at all.

\section{Disclosures}

Niels Fristrup MD PhD, Advisory Board Member, Pfizer inc.

GL: No relevant COls

Beyond this, authors declare that no financial support or other benefits from commercial sources have been received and none of the authors has any financial or otherwise competing interests that could create any conflict of interests.

\section{Funding}

The authors received no financial support for the research, authorship, and/or publication of this article.

\section{References}

1. Dale DC and Bolyard AA (2017) An update on the diagnosis and treatment of chronic idiopathic neutropenia Curr Opin Hematol 24(1) 46-53 https://doi.org/10.1097/MOH.0000000000000305 PMCID: 5380401

2. Palmblad J, Dufour C, and Papadaki HA (2014) How we diagnose neutropenia in the adult and elderly patient Haematologica 99(7) 1130-1133 https://doi.org/10.3324/haematol.2014.110288 PMID: 24986874 PMCID: 4077072

3. Klastersky J, de Naurois J, and Rolston K, et al. (2016) Management of febrile neutropaenia: ESMO Clinical Practice Guidelines Ann Oncol 27(suppl 5) v111-v118 https://doi.org/10.1093/annonc/mdw325 PMID: 27664247

4. Jin S, Pazdur R, and Sridhara R (2017) Re-evaluating eligibility criteria for oncology clinical trials: analysis of investigational new drug applications in $2015 \mathrm{~J}$ Clin Oncol 35(33) 3745-3752 https://doi.org/10.1200/JCO.2017.73.4186 PMID: 28968168 PMCID: 5692723

5. Schettini F, De Santo I, and Rea CG, et al (2018) CDK 4/6 inhibitors as single agent in advanced solid tumors Front Oncol 8 608 https:// doi.org/10.3389/fonc.2018.00608

6. Knudsen ES and Witkiewicz AK (2017) The strange case of CDK4/6 inhibitors: mechanisms, resistance, and combination strategies Trends Cancer 3(1) 39-55 https://doi.org/10.1016/j.trecan.2016.11.006 PMID: 28303264 PMCID: 5347397

7. Andersen CL, Tesfa D, and Siersma VD, et al (2016) Prevalence and clinical significance of neutropenia discovered in routine complete blood cell counts: a longitudinal study J Intern Med 279(6) 566-575 https://doi.org/10.1111/joim.12467 PMID: 26791682

8. Hsieh MM, Everhart JE, and Byrd-Holt DD, et al (2007) Prevalence of neutropenia in the U.S. population: age, sex, smoking status, and ethnic differences Ann Intern Med 146(7) 486-492 https://doi.org/10.7326/0003-4819-146-7-200704030-00004 PMID: 17404350

9. Newburger PE and Dale DC (2013) Evaluation and management of patients with isolated neutropenia Semin Hematol 50(3) 198-206 https://doi.org/10.1053/j.seminhematol.2013.06.010 PMID: 23953336 PMCID: 3748385

10. Gupta A and Dhingra A (2017) Incidental chronic neutropenia in an asymptomatic adult Cureus 9(10) e1779 PMID: 29255658 PMCID: 5732009

11. Capsoni F, Sarzi-Puttini P, and Zanella A (2005) Primary and secondary autoimmune neutropenia Arthritis Res Ther 7(5) 208-214 https:// doi.org/10.1186/ar1803 PMID: 16207350 PMCID: 1257445

12. Bray F, Ferlay J, and Soerjomataram I, et al (2018) Global cancer statistics 2018: GLOBOCAN estimates of incidence and mortality worldwide for 36 cancers in 185 countries CA Cancer J Clin 68(6) 394-424 https://doi.org/10.3322/caac.21492 PMID: 30207593 
13. Twelves C, Wong A, and Nowacki MP, et al (2005) Capecitabine as adjuvant treatment for stage III colon cancer N Engl J Med 352(26) 2696-2704 https://doi.org/10.1056/NEJMoa043116 PMID: 15987918

14. Tomita N, Kunieda K, and Maeda A, et al (2019) Phase III randomised trial comparing 6 vs. 12-month of capecitabine as adjuvant chemotherapy for patients with stage III colon cancer: final results of the JFMC37-0801 study Br J Cancer 120(7) 689-696 https://doi. org/10.1038/s41416-019-0410-0 PMID: 30833647 PMCID: 6461756

15. Or Knudsen A, Schledermann D, and Nyvang GB, et al (2016) Trends in gynecologic cancer among elderly women in Denmark, 19802012 Acta Oncol 55(Suppl 1) 65-73 https://doi.org/10.3109/0284186X.2015.1115119

16. Pignata S, Scambia G, and Ferrandina G, et al (2011) Carboplatin plus paclitaxel versus carboplatin plus pegylated liposomal doxorubicin as first-line treatment for patients with ovarian cancer: the MITO-2 randomized phase III trial J Clin Oncol 29(27) 3628-3635 https://doi.org/10.1200/JCO.2010.33.8566 PMID: 21844495

17. Aapro MS, Bohlius J, and Cameron DA, et al (2011) 2010 update of EORTC guidelines for the use of granulocyte-colony stimulating factor to reduce the incidence of chemotherapy-induced febrile neutropenia in adult patients with lymphoproliferative disorders and solid tumours Eur J Cancer 47(1) 8-32 https://doi.org/10.1016/j.ejca.2010.10.013

18. Crawford J, Becker PS, and Armitage JO, et al (2017) Myeloid growth factors, version 2.2017, NCCN clinical practice guidelines in oncology J Natl Compr Canc Netw 15(12) 1520-1541 https://doi.org/10.6004/jnccn.2017.0175 PMID: 29223990

19. Dassen AE, Bernards N, and Lemmens VEPP, et al (2016) Phase Il study of docetaxel, cisplatin and capecitabine as preoperative chemotherapy in resectable gastric cancer World J Gastrointest Surg 8(10) 706-712 https://doi.org/10.4240/wjgs.v8.i10.706 PMID: 27830043 PMCID: $\underline{5081553}$

20. Franzese E, Centonze S, and Diana A, et al (2019) PARP inhibitors in ovarian cancer Cancer Treat Rev 73 1-9 https://doi.org/10.1016/j. ctrv.2018.12.002

21. Friedlander M, Banerjee S, and Mileshkin L, et al (2016) Practical guidance on the use of olaparib capsules as maintenance therapy for women with BRCA mutations and platinum-sensitive recurrent ovarian cancer Asia Pac J Clin Oncol 12(4) 323-331 https://doi. org/10.1111/ajco.12636 PMID: 27917619

22. Norton L (2014) Cancer log-kill revisited Am Soc Clin Oncol Educ Book 3-7 https://doi.org/10.14694/EdBook_AM.2014.34.3 PMID: 24857052

23. Gibson C and Berliner N (2014) How we evaluate and treat neutropenia in adults Blood 124(8) 1251-1258 quiz 1378 https://doi. org/10.1182/blood-2014-02-482612 PMID: 24869938 